\title{
Estrogen-mediated downregulation of AIRE influences sexual dimorphism in autoimmune diseases
}

\author{
Nadine Dragin, ${ }^{1,2,3,4}$ Jacky Bismuth, ${ }^{1,2,3,4}$ Géraldine Cizeron-Clairac, ${ }^{5}$ Maria Grazia Biferi, ${ }^{1,2,3,4}$ Claire Berthault,,${ }^{6,7}$ Alain Serraf, ${ }^{8}$ \\ Rémi Nottin, ${ }^{8}$ David Klatzmann, ${ }^{9}$ Ana Cumano, ${ }^{6}$ Martine Barkats, ${ }^{1,2,3,4}$ Rozen Le Panse, ${ }^{1,2,3,4}$ and Sonia Berrih-Aknin ${ }^{1,2,3,4}$ \\ ${ }^{1}$ Sorbonne Universités, UPMC University of Paris 06, Paris, France. IINSERM U974, Paris, France. ${ }^{3}$ CNRS FRE 3617, Paris, France. ${ }^{4}$ AIM, Institute of Myology, Paris, France. ${ }^{5}$ CNRS UMR 8162, \\ Le Plessis-Robinson, France. ${ }^{6}$ INSERM U668, Unit for Lymphopoiesis, Immunology Department, Pasteur Institute, Paris, France. Université Paris Diderot, Sorbonne Paris Cité, Cellule Pasteur, Paris, France. \\ ${ }^{8}$ Hôpital Marie Lannelongue, Le Plessis-Robinson, France. ${ }^{9}$ Assistance Publique - Hôpitaux de Paris (AP-HP), Pitié-Salpêtrière Hospital, Biotherapy, Paris, France.
}

\begin{abstract}
Autoimmune diseases affect $5 \%$ to $8 \%$ of the population, and females are more susceptible to these diseases than males. Here, we analyzed human thymic transcriptome and revealed sex-associated differences in the expression of tissue-specific antigens that are controlled by the autoimmune regulator (AIRE), a key factor in central tolerance. We hypothesized that the level of AIRE is linked to sexual dimorphism susceptibility to autoimmune diseases. In human and mouse thymus, females expressed less AIRE (mRNA and protein) than males after puberty. These results were confirmed in purified murine thymic epithelial cells (TECs). We also demonstrated that AIRE expression is related to sexual hormones, as male castration decreased AIRE thymic expression and estrogen receptor $\alpha$-deficient mice did not show a sex disparity for AIRE expression. Moreover, estrogen treatment resulted in downregulation of AIRE expression in cultured human TECs, human thymic tissue grafted to immunodeficient mice, and murine fetal thymus organ cultures. AIRE levels in human thymus grafted in immunodeficient mice depended upon the sex of the recipient. Estrogen also upregulated the number of methylated $\mathrm{CpC}$ sites in the AIRE promoter. Together, our results indicate that in females, estrogen induces epigenetic changes in the AIRE gene, leading to reduced AIRE expression under a threshold that increases female susceptibility to autoimmune diseases.
\end{abstract}

\section{Introduction}

Autoimmune diseases, a group of 70 different diseases, represent the fifth leading cause of death by disease among females of reproductive age $(1,2)$. As suggested by several studies, various components, such as genetic predisposition (sexual chromosomes, epigenetics, microchimerism, parental inheritance), hormonal factors, and environmental exposure, could underlie sexual dimorphism in autoimmune diseases. Sex hormones have been shown as main factors in disease triggering and development (3, 4). The role of hormones has been described in the effector phase of autoimmune response, but not in the predisposition phase (5). For example, in systemic lupus erythematosus, estrogen favors survival of autoreactive B cells and skews their maturation toward a marginal zone phenotype (6). More generally, sex hormones play a role in the regulation of the balance of Th1/Th2 cytokines, with females more likely to develop a Th1 response, except during pregnancy, when Th2 response is predominant (7).

Autoimmunity is the result of tolerance breakdown, a phenomenon occurring essentially in the thymus, the site of $\mathrm{T}$ cell education. In the thymus, $\mathrm{T}$ cells undergo positive and negative selections in contact with stromal cells. T cells expressing TCR that recognize the MHC/self-peptide molecule with high avid-

\section{Related Commentary: p. 1239}

Authorship note: J. Bismuth and G. Cizeron-Clairac contributed equally to this work. Conflict of interest: The authors have declared that no conflict of interest exists. Submitted: March 25, 2015; Accepted: January 21, 2016.

Reference information: / Clin Invest. 2016;126(4):1525-1537. doi:10.1172/JCI81894. ity are deleted by apoptosis. Cells escaping this negative selection are potentially autoreactive and are involved in autoimmune disease development (8). The autoimmune regulator (AIRE), a transcription regulator expressed in medullary thymic epithelial cells (mTECs), is a key factor in the central tolerance and negative selection of autoreactive T cells. AIRE regulates the expression of tissue-specific antigens (TSAs). It has been involved in mTEC development and differentiation and could also induce a specific population of naturally occurring T-regulatory lymphocytes (Tregs) (9). The link between AIRE expression and autoimmune diseases has been demonstrated in humans and in animal models. In humans, mutations in the AIRE gene are responsible for an autosomal monogenic autoimmune defect called autoimmune polyendocrinopathy candidiasis ectodermal dystrophy (APECED). APECED patients display variable phenotype symptoms $(10,11)$ characterized by a combination of endocrine autoimmune diseases, such as Addison's disease, hypoparathyroidism, and type 1 diabetes. Mice deficient for Aire show high susceptibility to autoimmune diseases and increased autoreactive $\mathrm{T}$ cells in the periphery (12-14). Autoimmune destruction with cell infiltrates in peripheral organs is also found in mice deficient for the AIRE protein $(12,15,16)$.

Since the thymus is the site of central tolerance, we assumed that a comparison of the thymic transcriptome of males and females would reveal some secrets about potential differences in tolerance mechanisms that could shed light on increased female susceptibility to autoimmune diseases. This analysis of transcriptome indeed revealed that AIRE-dependent TSAs were more expressed in males than in females, raising the hypothesis that 

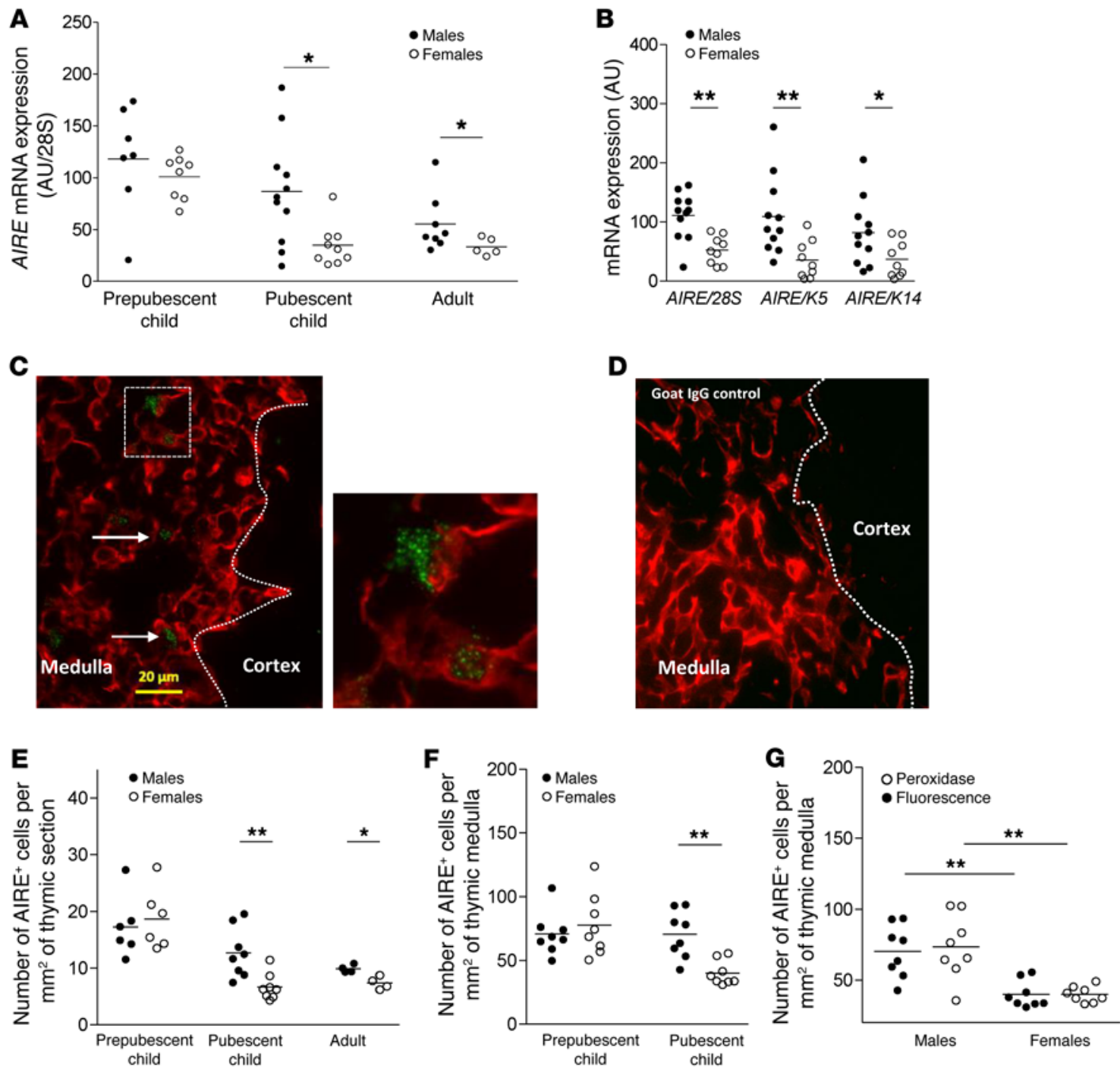

Figure 1. Differential AIRE expression in normal thymuses according to sex. AIRE mRNA levels, normalized to 285 , in 48 human thymuses during the aging process (A). The prepubescent child group (5 months to 11 years old) included 8 females and 7 males; the pubescent child group (13 to 17 years old) included 9 females and 11 males; the adult group (over 18 years old) included 5 females and 8 males. AIRE mRNA levels, normalized to 28S, K5, and K14 in thymuses of pubescent child (B). Representative pictures of a human thymus labeled with an anti-K14 antibody (red) and with an anti-AIRE antibody (green) (C) or purified goat IgG control (D). Arrows indicate AIRE ${ }^{+}$cells. Images were acquired with a Zeiss Axio Observer Z1 inverted microscope. Number of cells expressing AIRE protein per $\mathrm{mm}^{2}$ of thymic total section (E) or thymic medullary region (F) of human thymuses ( $n=4-8$ thymuses per sex and age). Compared analysis by immunohistochemistry and immunofluorescence of number of cells expressing AIRE protein per $\mathrm{mm}^{2}$ of thymic medulla of pubescent child (C). $P$ values were obtained using the nonparametric Mann-Whitney $U$ test. ${ }^{*} P<0.05 ;{ }^{*} P<0.01$.

AIRE is a key factor in female susceptibility to autoimmune disorders. By analyzing in detail AIRE expression in human and mouse thymuses as a function of sex and age, its regulation by hormones, and the link between AIRE expression levels and mouse susceptibility to autoimmune diseases, we validated our hypothesis.

\section{Results}

AIRE is less expressed in female than in male thymuses. We compared the human thymic transcriptome of males and females and observed differences in TSA expression (Supplemental Figure 1 and Supplemental Table 1; supplemental material available online with this article; doi:10.1172/JCI81894DS1). Since the expression of these TSAs is regulated by AIRE, we hypothesized that AIRE could be differentially expressed in males and females. Real-time
PCR performed on a total of 48 human thymuses, including prepubescent child, pubescent child, and adult samples, revealed that as humans aged, AIRE expression decreased substantially. However, the time curves were different for females compared with males, with strong decreased expression of AIRE in females at pubescence (Figure 1A). Expression of AIRE was normalized to the whole amount of RNA (28S), and to keratin 5 (K5) and keratin 14 (K14), the main keratins of the thymic medulla. The difference between pubescent males and females was significant no matter what gene was used for normalization (Figure 1B). We then analyzed AIRE protein expression in thymic human sections by immunofluorescence and immunohistochemistry. We chose the D17 anti-AIRE antibody that has already been validated and characterized in several articles in immunohistochemistry and flow cytometry studies 
A

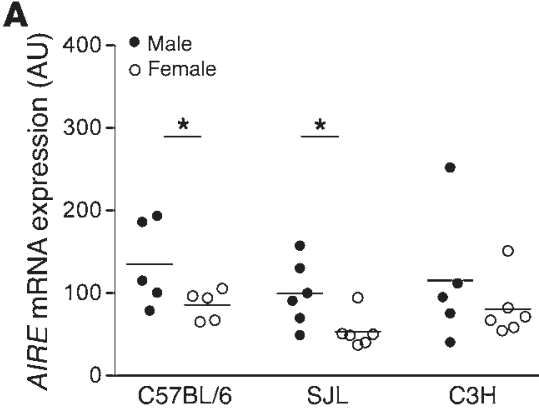

B

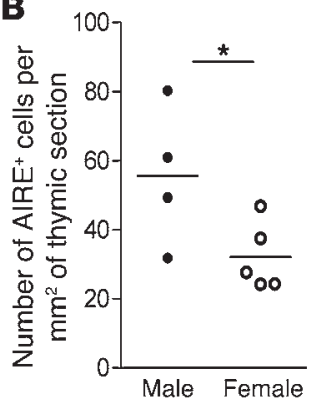

C

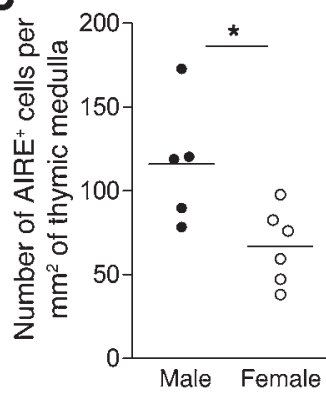

D

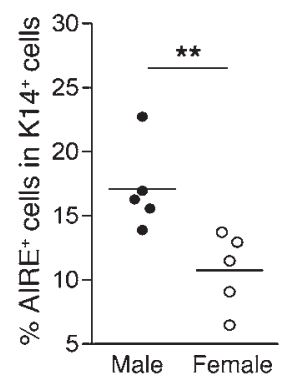

E

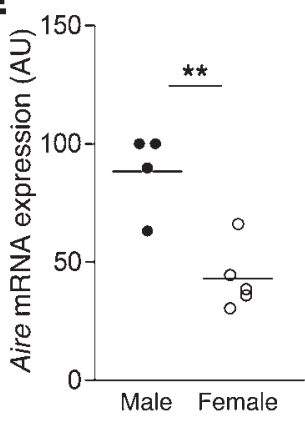

$\mathbf{F}$

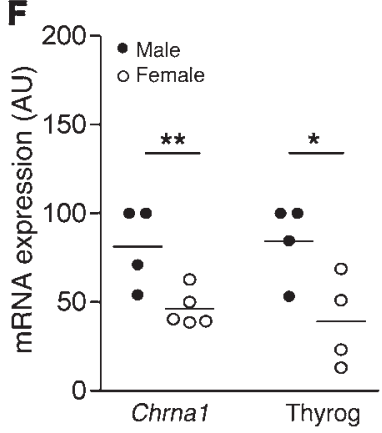

${ }^{\circ}$

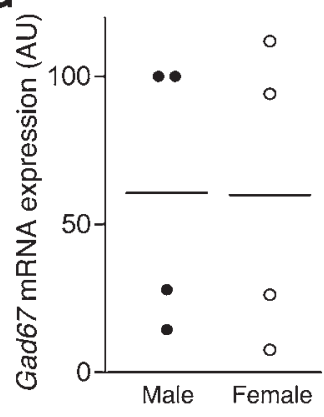

H

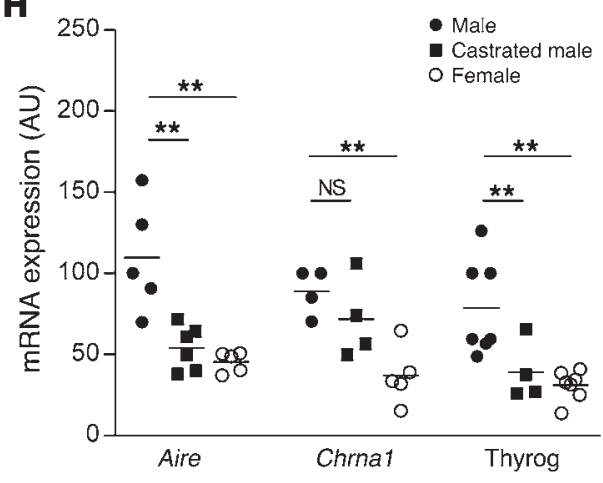

I

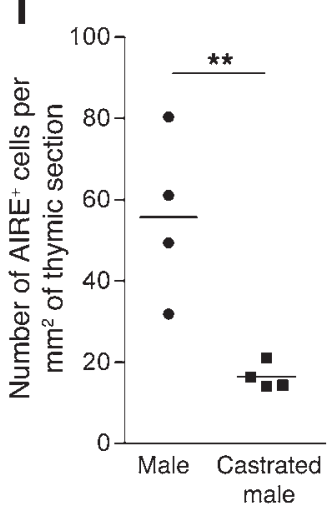

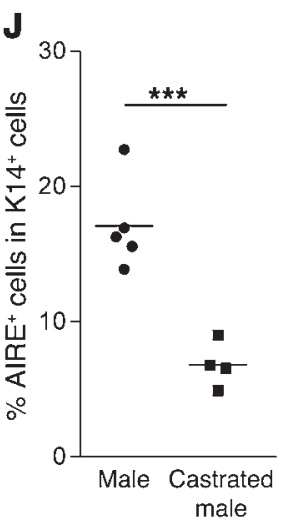

Figure 2. Differential AIRE and TSA expression in normal and castrated mouse thymuses and sorted mTECs. Aire mRNA expression in male and female mouse thymuses, 6 to 8 weeks old, in 3 different mouse strains (C57BL/6, SJL, and C3H) $n=5-6$ thymuses for each sex and strain. (A). Number of AIRE ${ }^{+}$ cells per $\mathrm{mm}^{2}$ of thymic sections (B), number of AIRE ${ }^{+}$cells per $\mathrm{mm}^{2}$ of thymic medulla (C), and percentage of cells expressing the AIRE protein in K14 ${ }^{+}$ in the thymic medullary area (D) of SJL mice aged of 6 to 8 weeks old by sex ( $n=4-6$ thymuses for each sex). mRNA expression of Aire (E), Chrna1 and thyroglobulin (thryog), 2 AIRE-dependent TSAs (F), and Gad67, an AIRE-independent TSA (C), in sorted mTECs from 6-week-old male and female C57BL/6 mice ( $n>4$ thymuses per sex). Thymic mRNA levels of Aire, Chrna1, and thyroglobulin $(\mathbf{H})$, number of AIRE-expressing cells per thymic section (I), and percentage of cells expressing the AIRE protein among K14+ cells in the thymic medullary area (J) of control and castrated SJL male mice $(n=4-6$ thymuses per group). Aire mRNA levels were normalized to K5. $P$ values were obtained using the nonparametric Mann-Whitney $U$ test. For $\mathbf{H}, P$ values were obtained using the 1-way ANOVA test. ${ }^{*} P<0.05 ;{ }^{* *} P<0.01 ;{ }^{* *} P<0.001$.

(17-19). Our observations are in agreement with previous studies showing that AIRE expression is primarily restricted to medullary areas (ref. 12 and Figure 1C). The staining, limited to the nucleus of some epithelial cells, had the typical nuclear dot pattern (Figure 1C). The specificity of the AIRE staining was confirmed with a purified goat IgG that did not show labeling (Figure 1D). The protein expression profile at different ages (prepubescent child, pubescent child, adult) was very similar to that observed for mRNA (Figure $1 \mathrm{E})$. Pubescent females had fewer $\mathrm{AIRE}^{+}$cells than males regardless of the factor of normalization used, the surface of the whole thymic section (Figure 1E), the surface of the thymic medulla (Figure $1 \mathrm{~F}$ ), or the $\mathrm{K}^{+} 4^{+}$cell number (Supplemental Figure 2). Further- more, analysis of AIRE protein expression in pubescent thymus was replicated using immunoperoxidase staining instead of immunofluorescence, with other sections from the same samples (8 males and 8 females). The results, expressed as number of cells per $\mathrm{mm}^{2}$ of thymic medulla (Figure $1 \mathrm{G}$ ), confirmed the first observation. Detailed numbers of cells are given in Supplemental Table 2. Of note, interindividual variability was much higher at the mRNA level than at the protein level (Supplemental Table 3), which is likely a consequence of mRNA regulation or clearance and the high stability of protein (commonly observed for other genes; ref. 20).

Aire is less expressed in female than male mouse thymuses. We wondered whether these changes in AIRE expression during 


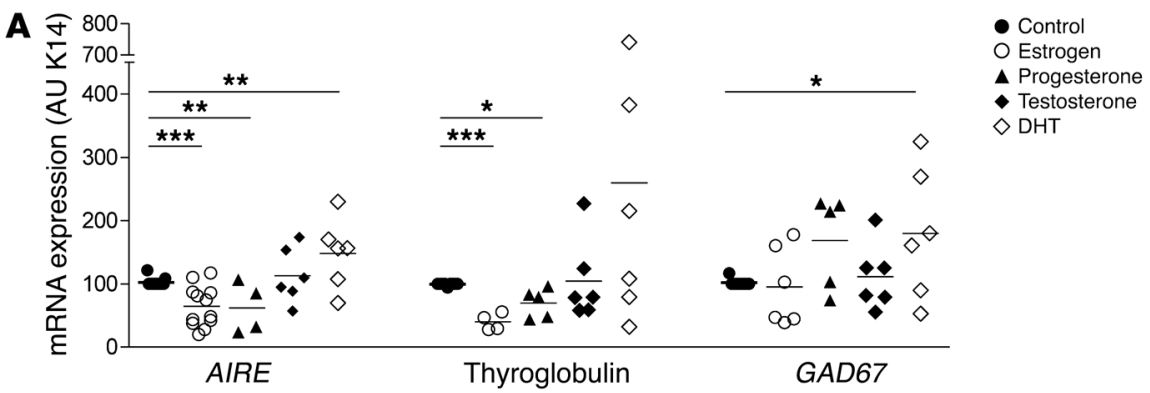

Figure 3. Influence of sex hormones on AIRE and TSA expression in cultured human TECs. Effects of estrogen, testosterone, progesterone, and DHT (1 $\mathrm{nM}$ ) on the mRNA expression of AIRE, AIRE-dependent TSA (thyroglobulin), and AIRE-independent TSA gene (GAD67) in human primary TECs (A). Dose-effect of estrogen (B) and DHT (C) on the mRNA expression of $A I R E$, thyroglobulin, and GAD67 in human primary TECs. Dose effect of DHT in the presence of estrogen $(1 \mathrm{nM})$ on the mRNA expression of AIRE in human primary TECs (D). Dose effect of estrogen in the presence of DHT $(1 \mathrm{nM})$ on the mRNA expression of AIRE in human primary TECs (E). $n>4$ individual human thymuses. Results are means \pm SEM in $\mathbf{B}$ and $\mathbf{C}$. ${ }^{*} P<0.05$; ${ }^{* *} P<0.01$; ${ }^{* *} P<0.005$ to $0.001,1$-way ANOVA.
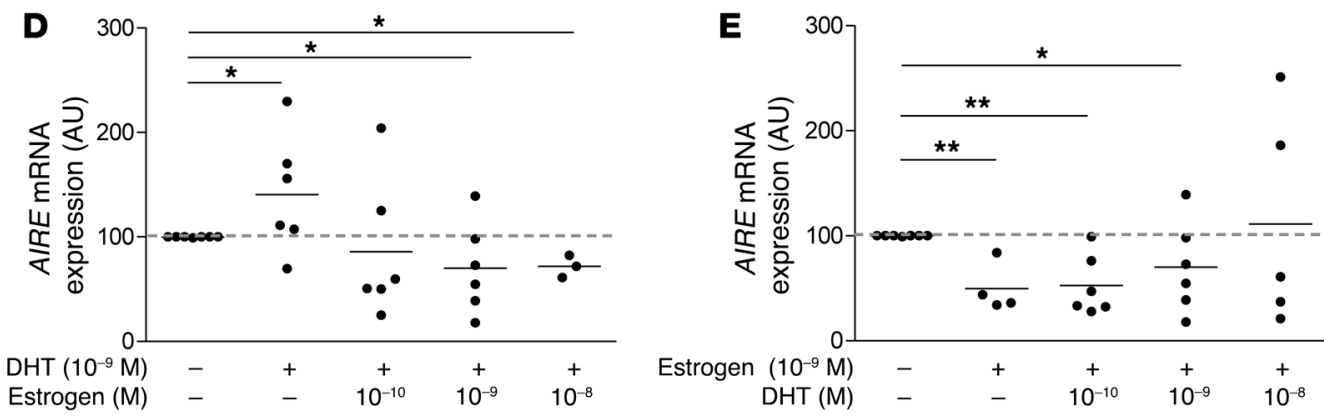

human aging also occurred in mice. Therefore, we extended mRNA analysis to various mouse strains known to show sexual dimorphism or susceptibility toward experimental autoimmune diseases. Interestingly, C57BL/6 and SJL females, which are more susceptible to autoimmune diseases compared with males $(21,22)$, expressed less Aire than their male counterparts (Figure 2A). Conversely, in $\mathrm{C} 3 \mathrm{H}$ mice, which do not display autoimmune disease susceptibility (23) the differences in Aire mRNA levels between males and females was not significant (Figure 2A).

To confirm these results at the protein level, we counted the number of AIRE-expressing cells in the thymus sections of male and female SJL and $\mathrm{C} 3 \mathrm{H}$ mice. The number of $\mathrm{AIRE}^{+}$cells was higher in adult SJL males compared with females, irrespective of the normalization used, whole thymus section surface (Figure 2B), thymic medulla surface (Figure 2C), or the number of medullary $\mathrm{K} 14^{+}$cells (Figure 2D). Detailed numbers of AIRE-positive cells are given in Supplemental Table 4. Representative medullary areas of 3 male and female mouse thymuses are shown in Supplemental Figure 3. In $\mathrm{C} 3 \mathrm{H}$ mice, the number of $\mathrm{AIRE}^{+}$cells was not different in males compared with females, irrespective of the normalization used, whole thymus section surface, or thymic medullary surface (Supplemental Figure 4).

In order to validate that the low level of Aire mRNA expression in female whole thymuses was due to a decreased expression in medullary TECs, we isolated and FACS sorted mTECs by selecting the cells with a $\mathrm{CD} 45^{-} \mathrm{MHCII}^{+} \mathrm{UEA}^{+}$ly51- phenotype, as previously described in the literature (refs. 24, 25, and Supplemental Figure 5). mRNA expression of Aire was lower in female compared with male sorted mTECs (Figure 2E). To validate this lower expression of Aire in females, we also analyzed the expression of 2 TSAs known to be regulated by AIRE, acetylcholine receptor alpha (Chrna1) (26-28) and thyroglobulin (29, 30). These AIRE-dependent TSAs were also significantly decreased in female mTECs compared with male mTECs (Figure $2 \mathrm{~F}$ ). In contrast, AIRE-independent TSA, glutamate decarboxylase 67 (Gad67) (refs. 31, 32, and Figure 2G), was not differently expressed in male or female mouse mTECs, validating our observations of whole thymuses. These results raised the hypothesis of the involvement of sexual hormones in thymic AIRE expression.

Therefore, we investigated the possibility of modulating Aire expression in vivo by modifying hormone levels. We proceeded by bilaterally castrating SJL male mice and analyzing thymic Aire expression 2 weeks after surgery. In castrated SJL male mice, we observed a significant decrease in Aire, Chrnal, and thyroglobulin mRNA expression (Figure $2 \mathrm{H}$ ) as well as in the number of $\mathrm{AIRE}^{+}$cells per thymic section (Figure 2I) or normalized to K14-positive cells (Figure 2J).

Male castration has been reported by various authors to affect thymic cellularity (33) and to regenerate involuted thymus, hence reversing age-related thymic atrophy $(34,35)$. We observed in 


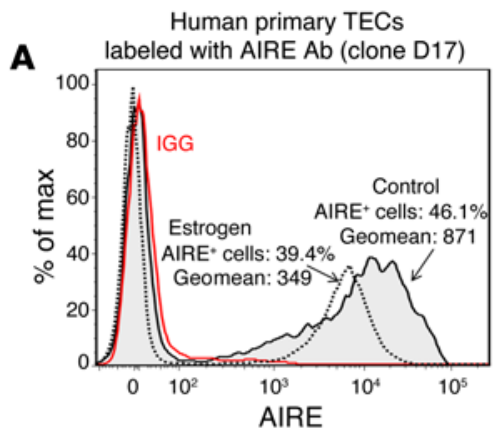

B

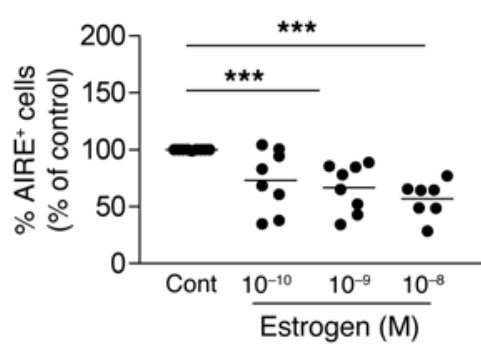

C

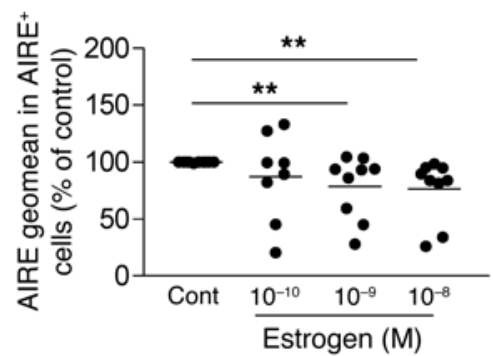

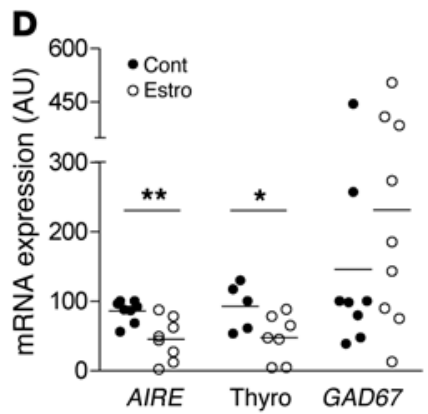

E

$\mathbf{F}$
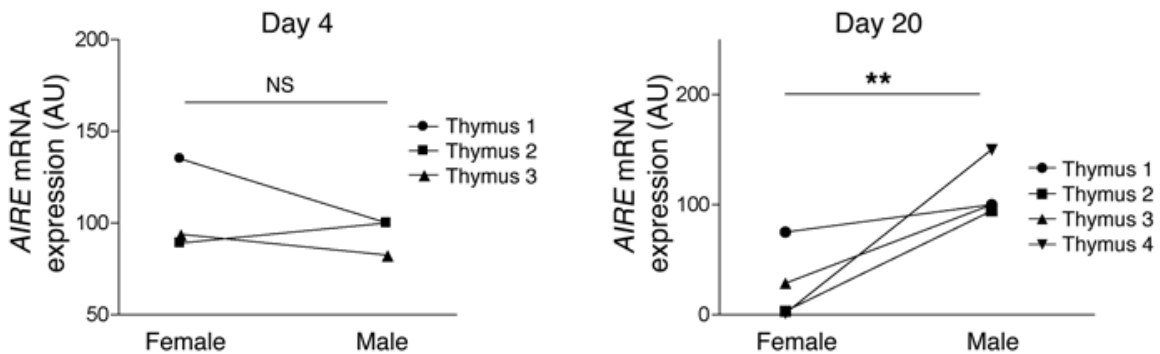

G

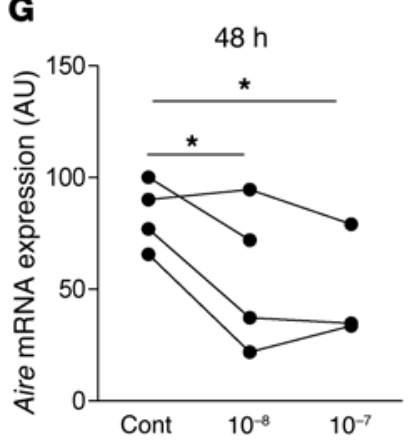

H

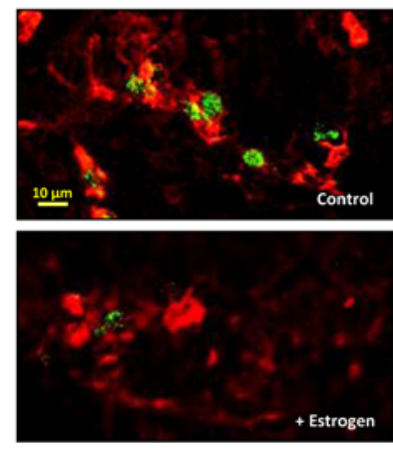

I

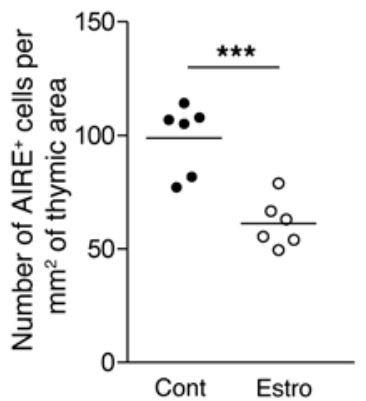

Figure 4. Influence of estrogen on AIRE and TSA expression in cultured human TECs, human thymic tissue grafted to NSG mice, and mouse fetal thymic organ culture. Representative flow cytometry plot of estrogen effect (1 nM) on AIRE protein level in human primary TECs; the control isotype is shown (red histogram) (A). Effect of estrogen on the percentage of AIRE-positive cells (B) and AIRE fluorescence geometric mean (geomean) (C) in cultured human primary TECs. For each experiment, control untreated cells were normalized to $100(n=8-9)$. Effect of daily intraperitoneal injections of estrogen (200 $\mu \mathrm{g})$ on AIRE, thyroglobulin, and GAD67 mRNA expression in human thymic tissue grafted to NSG female mice aged of 5 to 6 weeks $(n=8)(D)$. Effect of the mouse sex recipient on AIRE mRNA expression in human thymic tissue engrafted subcutaneously in NSG mice for 4 days (E) ( $n=3$ individual human thymuses) and 20 days (F) ( $n=4$ individual human thymuses). Effect of estrogen on the mRNA expression of Aire, in mouse fetal thymic organ cultures (G). Each point represents the mean value of an experiment using thymuses from 4 to 5 different fetuses. Representative picture of mouse FTOC labeled with anti-K14 (red) and anti-AIRE (green) antibodies. Images were acquired with a Zeiss Axio Observer Z1 inverted microscope (H). Effect of estrogen (estro) (10 $\left.{ }^{-7} \mathrm{M}\right)$ on the number of cells expressing AIRE protein in the mouse FTOC (I). Cont, control. $P$ values were obtained using the 1-way ANOVA test (B and $\mathbf{C}$ ), nonparametric Mann-Whitney $U$ test for unpaired data $(\mathbf{D}$ and $\mathbf{I})$, and the Wilcoxon test for paired data $(\mathbf{E}-\mathbf{G}) .{ }^{*} P<0.05 ;{ }^{* *} P<0.01 ;{ }^{* * *} P<0.005$ to 0.001 .

male castrated thymuses, as previously reported in the literature $(36,37)$, a significant reduction in the medullary area (Supplemental Figure 6A). However, the number of medullary epithelial cells $\left(\mathrm{K} 14^{+}\right)$was unchanged in the whole thymus section (Supplemental Figure 6B). Therefore, the decrease in Aire expression in castrated male mice was not due to the reduction of the medullary areas, nor to the reduction of mTEC number, but was likely due to a true diminution of Aire expression.

In female SJL mice, ovariectomy induced a slight increase in Aire mRNA expression (Supplemental Figure 6C), while AIRE protein levels were unchanged compared with those of control female mice (Supplemental Figure 6, D and E). Female castration induced changes in thymic global size compared with male castration, but led to subtle effects on AIRE expression, possibly due to estrogen production by the adrenal gland and fat tissue, which may maintain estrogen in the mouse serum $(38,39)$.

Estrogen decreases AIRE expression in cultured human and mouse TECs. These results suggest that sex hormones could regulate thymic AIRE expression in vivo. To investigate this hypothesis, we analyzed the in vitro effects of the main sexual hormones on AIRE and TSA gene expression in well-defined and validated primary human TECs from thymic explants, known to be enriched in mTECs (40, 41), as shown in Supplemental Figure 7. Human TECs were subcultured and treated with estrogen, testosterone, dihydrotestosterone 
A

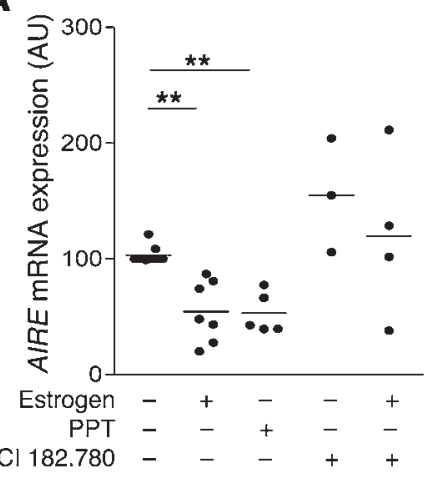

B

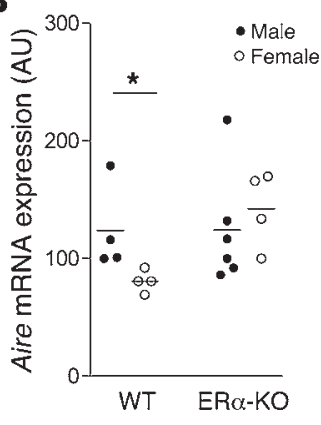

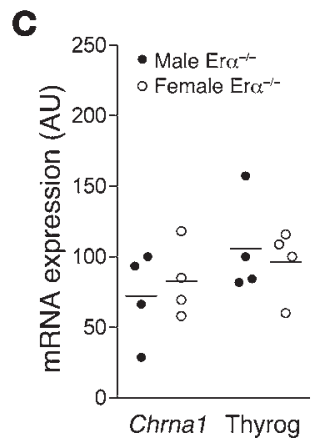

D

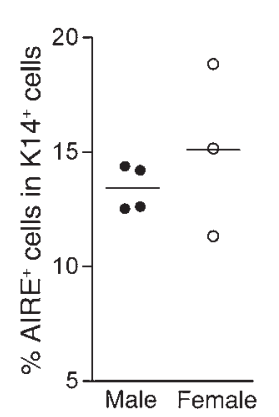

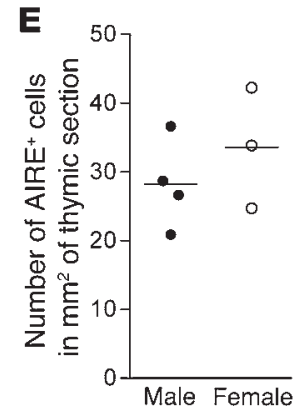
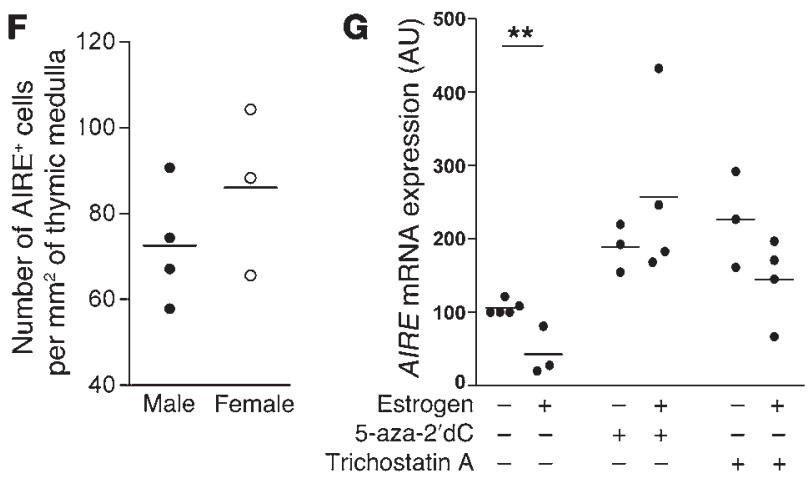

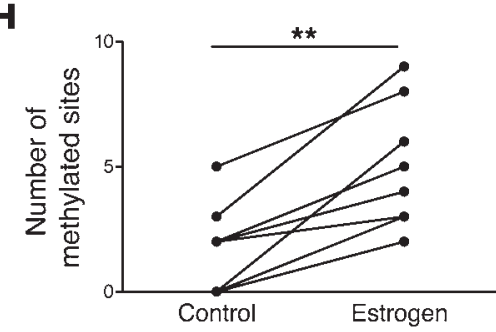

I

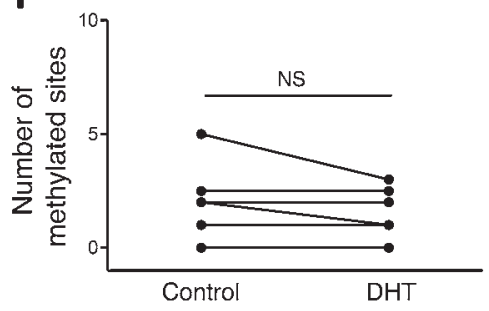

J

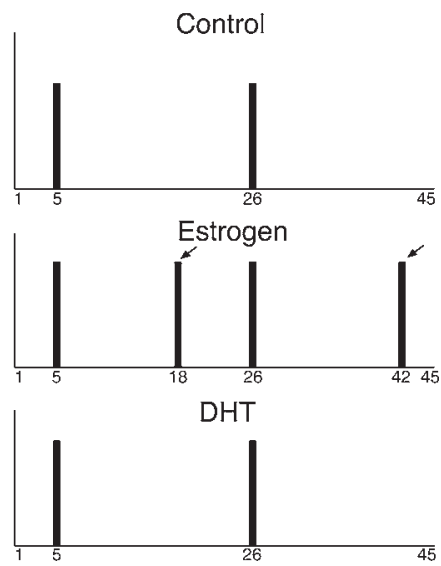

Figure 5. Pathways involved in estrogen-induced downmodulation of thymic AIRE expression. In vitro effects of the estrogen agonist PPT (10 nM) and the ER antagonist ICI $182.780(20 \mu \mathrm{M})$ on AIRE mRNA expression in TECs $(n=3-7)$ (A). Aire (B) and AIRE-dependent TSA (Chrna1, and thyroglobulin) (C) mRNA expression in male and female thymuses of ER $\alpha$-KO mice $(n=4-6)$. Percentage of cells expressing the AIRE protein among K14+ cells (D), number of AIRE-expressing cells per $\mathrm{mm}^{2}$ of thymic section $(\mathbf{E})$ and per $\mathrm{mm}^{2}$ of thymic medulla $(\mathbf{F})$, of male and female ER $\alpha$-KO mice $(n=3-4)$. Effects of 5 -aza2'-deoxycytidine (5-aza-2'dC) (5 $\mathrm{MM})$, a DNA methylation inhibitor, and trichostatin A (50 nM), a histone deacetylase inhibitor on AIRE mRNA expression in TEC cultures (G) $(n=3-4)$. Effect of estrogen ( $1 \mathrm{nM})(\mathbf{H})$ and DHT (1 nM) (I) on methylation site levels of CpG motifs in the human AIRE promoter in independent cultured human TECs (8 for estrogen and 6 for DHT). Representative methylation pattern of the CpG motifs within the human AIRE gene promoter in primary cultured TECs treated with $1 \mathrm{nM}$ of estrogen or of DHT (J). Each bar represents the methylation of a single $\mathrm{CpG}$ motif. The arrows show methylation sites that appeared after estrogen treatment. $P$ values were obtained using the 1-way ANOVA test $(\mathbf{A}$ and $\mathbf{G})$, the Mann-Whitney $U$ test for unpaired data (B-F), and the Wilcoxon test for paired data (H and I). ${ }^{*} P<0.05$ to $0.01 ;{ }^{* *} P<0.001$.

(DHT) (the main testosterone active metabolite), and progesterone at a physiological concentration $\left(10^{-9} \mathrm{M}\right)$. Estrogen and progesterone markedly decreased mRNA expression of AIRE and AIREdependent TSA (thyroglobulin), but not of AIRE-independent TSA (GAD67). Even though testosterone induced no effect, DHT stimulated expression of AIRE (Figure 3A). Human TECs were then treated with estrogen and DHT at different concentrations. Estrogen induced a significant decrease in the mRNA expression levels of both AIRE and thyroglobulin (Figure 3B). GAD67 mRNA expression did not decrease, irrespective of the concentration of estrogen (Figure 3B). In contrast, DHT tended to increase both AIRE and TSA expression (Figure 3C). Moreover, we performed an analysis of combined treatments with estrogen and DHT. We observed that DHT at a low dose has no effect on the reduced expression of AIRE induced by estrogens, while at the highest dose $\left(10^{-8} \mathrm{M}\right)$, it increased AIRE expression (Figure 3D). Conversely, estrogen reduced the 
A

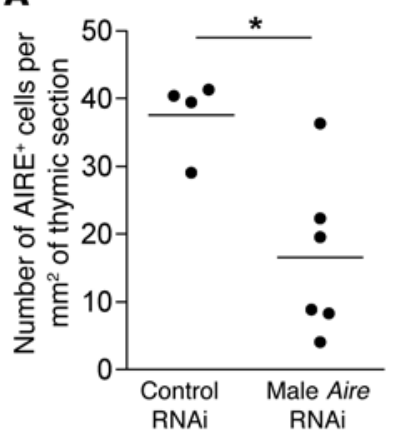

D

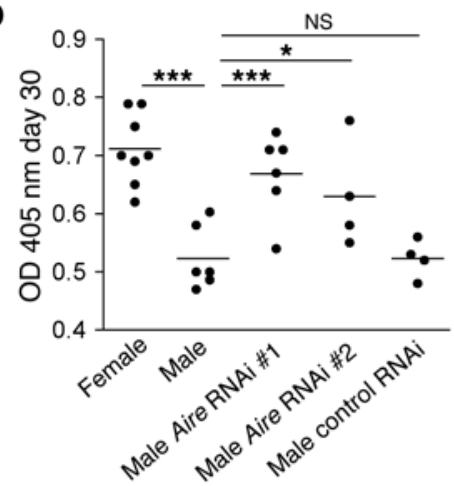

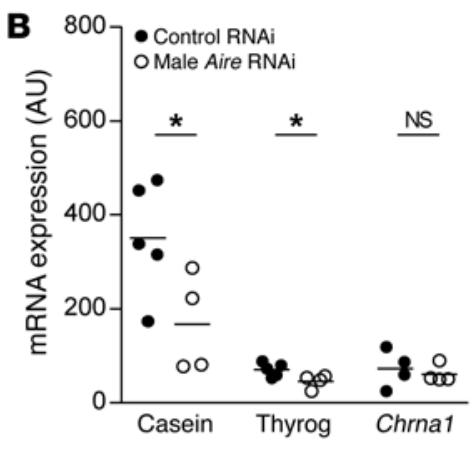

E

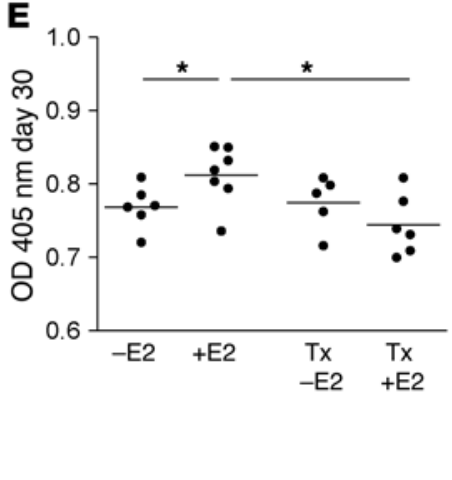

C

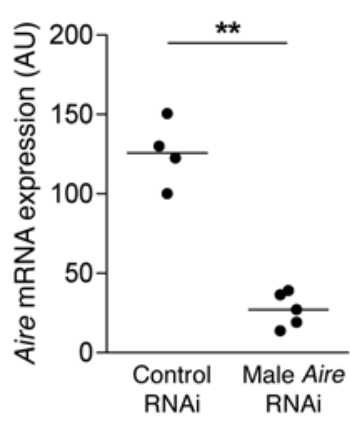

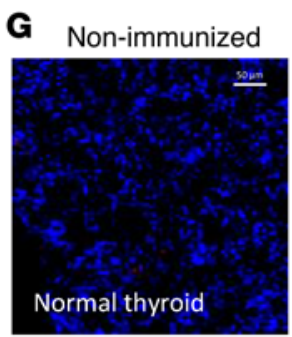
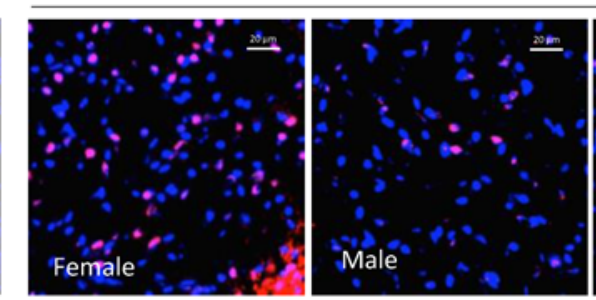

Immunized

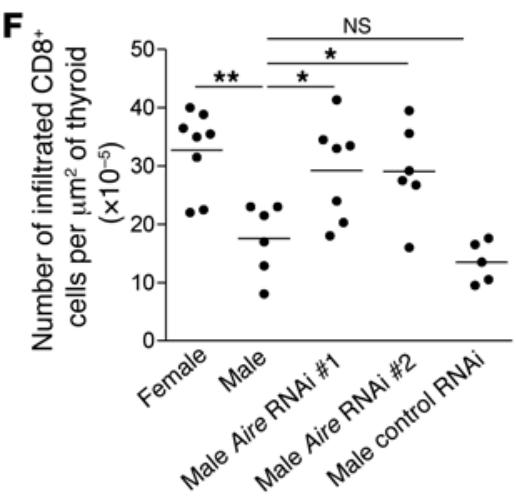

Figure 6. Modulation of AIRE thymic expression by AIRE-miRNAi and its effect on male susceptibility to EAT. Aire (A) and AIRE-dependent TSA (B) mRNA expression in SJL mouse thymuses at day 6 after scAAV9-miRNAi (850) injection $(n=4-5)$. Percentage of cells expressing the AIRE protein in the thymic section area (C) of SJL mouse after AIRE sCAAV9-miRNAi intrathymic injection $(n=4-6)$. SJL mice were immunized with $100 \mathrm{nmol}$ of p2340 in CFA emulsion by subcutaneous injections 7 days after intrathymic injection of 2 different AIRE scAAV9-miRNAi. Sera were collected at day 30 and the levels of antibodies to the thyroglobulin peptide p2340 in male, female, and AIRE scAAV9-miRNAi treated-male SJL mice were assessed by ELISA (D) ( $n=4-8$ sera per group). Bilaterally castrated SJL male mice were thymectomized (TX) and received a subcutaneous injection of $1 \mu \mathrm{g}$ of estradiol (E2) every 2 days, and were challenged for EAT. Sera were collected at day 30 , and the levels of antibodies to the thyroglobulin peptide p2340 were assessed by ELISA (E) ( $n=5-7$ sera per group). Number of infiltrating CD8-positive cells per $\mu \mathrm{m}^{2}$ of thyroid (F) ( $n=4-8$ mice per group). Representative photographs of mouse thyroid immunostaining labeled with an anti-CD8 antibody (red) of nonimmunized and immunized mice (G) for EAT challenge. Images were acquired with a Zeiss Axio Observer Z1 inverted microscope (F). $P$ values were obtained using the Mann-Whitney $U$ test $(\mathbf{A}-\mathbf{C})$ and the 1-way ANOVA test $(\mathbf{D}-\mathbf{F}) .{ }^{*} P<0.05 ;{ }^{* *} P<0.01 ;{ }^{* * *} P<0.001$.

overexpression of AIRE induced by DHT (1 nM) (Figure 3E), showing that estrogen and DHT have opposing effects on AIRE mRNA levels. When both hormones were added at $10^{-9} \mathrm{M}$, the dominant effect was that of estrogen. These results suggest that DHT in males favors an upregulation of AIRE thymic expression. However, in females, estrogen downregulates it.

Since estrogen is the primary hormone regulated in females during puberty - a period when AIRE expression is differentially expressed in human thymuses - these results prompted us to focus on estrogen effects on AIRE protein expression in TECs, engrafted human thymuses, and mouse fetal thymus organ cultures (FTOCs). We showed that, in primary human TECs, both the percentage of $\mathrm{AIRE}^{+}$cells and the fluorescence intensity of
AIRE expression in $\mathrm{AIRE}^{+}$cells were reduced by estrogen treatment (Figure 4, A-C). The decreased number of $\mathrm{AIRE}^{+}$cells may be explained by either a decreased level of AIRE under a threshold that makes it undetectable or a reduced differentiation toward $\mathrm{AIRE}^{+}$cells in the presence of estrogens.

The effects of estrogen were analyzed in human thymic fragments grafted in immunodeficient mice treated with estrogen (Figure 4D), and the results confirmed the estrogen downregulation effect observed in vitro. Furthermore, the impact of the sex of the mouse recipient on the modulation of AIRE expression levels was investigated with different human thymuses engrafted in male and female NOD/SCID/IL-2R $\gamma$-deficient (NSG) mice. Aire thymic mRNA levels were assessed at days 4 and 20. The sex of 


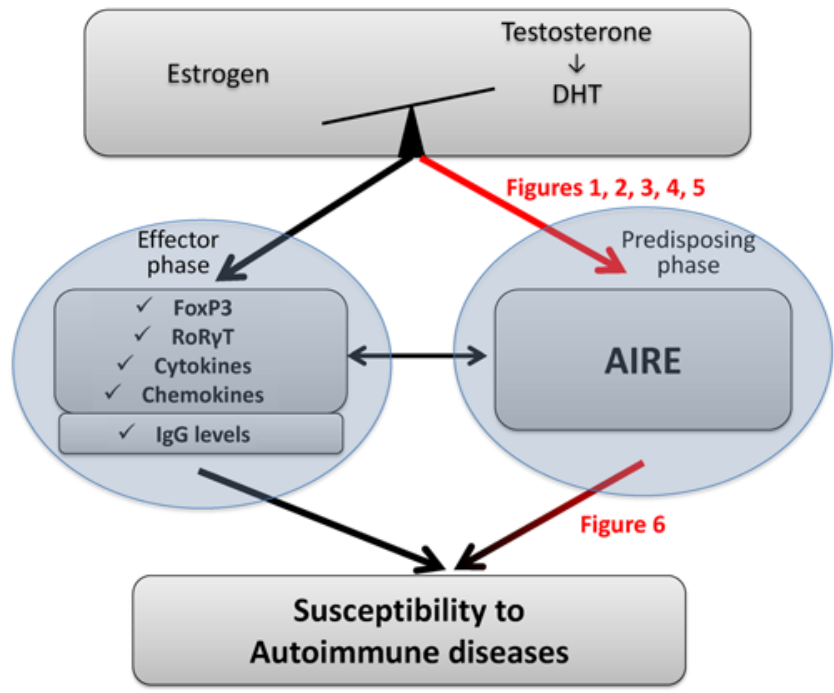

the thymus recipient significantly affected Aire expression levels at day 20, but not at day 4 (Figure 4, E and F). This late effect is likely related to the limited vascularization of the graft under the skin in the first week after graft.

Finally, in mouse FTOCs, estrogen also induced a decrease in Aire expression at both the mRNA level (Figure 4G) and the protein level (Figure 4, H and I). Typical AIRE-positive cells could be visualized on FTOC sections (Figure $4 \mathrm{H}$ ), and their cell numbers decreased in the presence of estrogens (Figure 4I). Altogether, these in vitro and in vivo data suggest that estrogen contributes substantially to the downregulation of AIRE and AIRE-dependent TSA expression in TECs.

Estrogen induces epigenetic changes in the AIRE promoter. Since estrogen is clearly involved in the regulation of AIRE in mouse and human TECs, we investigated its mechanism of action by studying the involvement of ER $\alpha$, the main ER isoform expressed in TECs (42). An ER $\alpha$ preferential agonist (PPT) mimicked estrogen effects on AIRE expression in human TECs. The ER $\alpha$ antagonist (ICI 182, 780) inhibited the effects of estrogen (Figure 5A). In vivo, $\mathrm{ER} \alpha-\mathrm{KO}$ (also known as $E s r 1^{-/-}$) female and male mice, with a C57BL/6 background, expressed the same levels of Aire and AIREdependent TSA thyroglobulin and Chrna1 (Figure 5, B and C). These results were confirmed at the protein level (Figure 5, D-F) irrespective of the factor used for normalization. As described in Yellayi et al., the size of the medulla is reduced in these ER $\alpha$ KO mice, similarly in males and females (43), suggesting that the similar AIRE expression in male and female ER $\alpha$-KO mice was not due to thymic structural changes (Supplemental Figure 8). These results indicate that estrogen regulated the thymic expression of AIRE through $\mathrm{ER} \alpha$ receptors.

No complete estrogen-responsive element has been found in the AIRE promoter. However, constitutive AIRE expression is known to be modulated through DNA methylation and/or histone acetylation modifications (44). Indeed, estrogen's regulation of AIRE was completely blocked by 5 -aza-2'-deoxycytidine, an inhibitor of DNA methylation (Figure $5 \mathrm{G}$ ). There was no change in AIRE regulation in the presence of trichostatin $\mathrm{A}$, an inducer of histone deacetylation (Figure $5 G$ ). These results suggest that the
Figure 7. Schematic representation of sexual hormone crosstalk effect on tolerance mechanisms involved in autoimmune disease. The balance between estrogen and testosterone has effects on many genes involved in the effector phase of autoimmune diseases, namely lgG, cytokines, and chemokines as well as transcription factors expressed in Tregs (FoxP3) and Th17 (RoR $\gamma \mathrm{T})$ cells. Our data show that the balance between hormones regulates AIRE expression, with estrogens decreasing its level (Figures 1-5). The level of AIRE affects the susceptibility to autoimmune diseases. This statement, which has largely been described in the literature, was confirmed here by specifically reducing the thymic expression of AIRE by intrathymic injection of AIRE-specific miRNAi (Figure 6). Red arrows indicate findings from this study.

regulation of $A I R E$ expression by estrogen was dependent upon DNA methylation, but not histone acetylation. In order to confirm this hypothesis, we sequenced the AIRE promoter in primary cultured human TECs treated with estrogen or DHT. The number of methylation sites significantly increased after estrogen treatment (Figure 5H), but was unchanged with DHT treatment (Figure 5I) compared with that in untreated cells. Analysis of AIRE methylation sites demonstrated that some methylation sites (site 4 or 5 ) were often found in primary cultured human TECs obtained from different human thymuses, while estrogen more frequently challenged the methylation of sites 32, 40, 41, or 42. Interestingly, an increased number of methylation sites with estrogen was observed in all TEC samples analyzed $(n=8)$. A representative example of estrogen and DHT treatments on AIRE promoter methylation status is presented in Figure 5J.

Reducing Aire thymic expression in male mice increases their susceptibility to EAT. By the use of AIRE-deficient models, a link between AIRE and autoimmunity has been clearly established (45). However, the influence of AIRE expression levels and female predisposition to autoimmunity remains unknown. We demonstrated in different in vivo and in vitro models that estrogen downregulated the expression of AIRE. We thus assume that AIRE downregulation by sexual hormones, including estrogen, may contribute to the increased female predisposition to autoimmune diseases. To investigate further this hypothesis and as a proof of concept, we used an experimental autoimmune thyroiditis (EAT) mouse model $(46,47)$ known to have a higher prevalence in females and castrated males (48) compared with normal males. Since male castration induced a significant decrease in Aire mRNA expression (Figure $2 \mathrm{H}$ ) and an increased male susceptibility to EAT (48), we wondered whether a link exists between these 2 observations. We chose to decrease Aire expression in the male thymus by expressing a specific artificial inhibitory microRNA (miRNAi) (49) using a self-complementary adeno-associated virus vector of serotype 9 (scAAV9) (50) instead of modulating estrogen levels that would also modulate several immune components, including cytokines and immunoglobulins (51-53). The efficiency of 3 miRNAi (Supplemental Table 5) in downregulating the expression of AIRE was 
first assessed in vitro (Supplemental Figure 9); we then selected the most efficient miRNAi (AIRE iRNA 850 and AIRE iRNA 288) and produced them in scAAV9 vectors. We investigated efficacy of AIRE iRNA 850 in vivo to reach thymic stromal cells (Supplemental Figure 10) and to downregulate thymic Aire expression by quantitative reverse-transcriptase PCR (qRT-PCR) analyses (Figure 6A). We also observed that AIRE-dependent TSAs, including thyroglobulin, were decreased in the thymus of miRNAi-treated mice (Figure 6B). In addition, the decreased number of $\mathrm{AIRE}^{+}$cells per surface of thymic section was observed by an immunohistochemistry analysis of the thymic sections (Figure 6C). Male mice injected with AIRE scAAV9-miRNA into the thymus displayed increased autoantibodies against thyroglobulin (Figure 6D).

To create an in vivo link among estrogen, AIRE, and autoimmune thyroiditis, we performed an experiment in which 4-weekold male mice were castrated, and we evaluated the effects of estrogens, in the presence or absence of the thymus, on autoimmune thyroiditis. We observed, as previously shown by Okayasu et al. (48), that estrogen increased the level of anti-thyroglobulin antibodies in nonthymectomized mice. However, when the mice were thymectomized, the effect of estrogen was abolished (Figure $6 \mathrm{E})$. These results clearly demonstrate that the deleterious effect of estrogen was linked to the thymus. Taking into account our previous demonstration that estrogen reduced AIRE expression, we clearly show that estrogen, by downregulating thymic AIRE expression, can favor the emergence of autoimmunity, an effect undetectable in the absence of thymus.

In a thyroiditis mouse model, the thyroid infiltrates are complex and include $\mathrm{CD}^{+}$and $\mathrm{CD}^{+} \mathrm{T}$ cells, $\mathrm{F} 4 / 80^{+}$macrophages, and $\mathrm{B} 22 \mathrm{O}^{+} \mathrm{B}$ cells (54). Thyroid cell infiltrations were analyzed with CD8 and F4/80 markers and counted as described by Caturegli et al. (55). The number of $\mathrm{CD}^{+}$infiltrating cells was lower in males compared with females, but increased CD8 ${ }^{+}$infiltrating cells were found in male mice treated with AIRE miRNAi compared with control miRNAi or with control male mice (Figure 6 , F and G). Similar results were obtained when using the F4/80 marker (Supplemental Figure 11). These results indicate that the specific thymic decrease in AIRE expression in males made them similar to the females in regard to the development of EAT. These findings validate the hypothesis that low expression of Aire may influence female autoimmune diseases susceptibility.

\section{Discussion}

Our data demonstrate the critical role that estrogen plays in AIRE thymic expression (mRNA and protein) and the consequences in terms of autoimmune disease susceptibility. Our results shed light on why most autoimmune diseases occur after puberty in females, when estrogen levels increase and contribute a new piece to the autoimmune disease predisposition puzzle (Figure 7).

AIRE, TSAs, and tolerance. The thymus, the primary organ involved in lymphocyte education, plays a major role in the development of immune central tolerance. AIRE is a transcription regulator expressed by mTECs that is essential for the induction of central tolerance and acts by regulating TSA expression. There is strong evidence that AIRE deficiencies result in altered AIREdependent TSA expression that leads to perturbed negative selection of autoreactive thymocytes. One potential mechanism, not yet proved in vivo, has been suggested. This mechanism assumes that AIRE induces mTEC apoptosis and favors crosspresentation of TSA proteins present in apoptotic cells to thymic dendritic cells (56). In addition, AIRE has been shown to have additional functions, as it is involved in mTEC differentiation and maturation, chemokine production, and Treg induction (9).

So far, deficient mouse models have provided clear information on the role of Aire in central tolerance. Aire ${ }^{-/-}$mice develop cell infiltrates in peripheral tissues, associated with deposition of autoantibodies. In several models involving AIRE-dependent TSA, Aire ${ }^{-/-}$mice were more susceptible to spontaneous (57) or induced autoimmune diseases than WT mice $(58,59)$. Furthermore, Aire-deficient mice had Tregs that failed to prevent spontaneous experimental colitis (60).

Of course, not all TSAs are regulated by AIRE and not all autoimmune diseases are due to AIRE-dependent TSAs. Nevertheless, in several models, it appears that AIRE-deficient mice have an increased susceptibility to autoimmunity, even when the TSAs are AIRE-independent, suggesting the involvement of the additional functions of AIRE discussed above. In experimental autoimmune encephalomyelitis (EAE), a model induced by proteolipid protein (PLP) or myelin oligodendrocyte glycoprotein (MOG), AIRE-independent TSAs, Aharoni et al. demonstrated that AIRE-deficient mice display defective Tregs that participate in induced autoimmune reactions (61). Kurisaki et al. reported that Aire $^{-/}$mice showed significant lymphocyte infiltration in the pancreas and stomach due to AIRE-independent autoantibody production (Pdia2) (62) suggesting that AIRE may regulate the survival of autoreactive $\mathrm{T}$ cells beyond transcriptional control of self-protein expression in the thymus (63). These studies highlight that the lack of AIRE results in high susceptibility to spontaneous and induced autoimmune diseases linked to autoreactive $\mathrm{T}$ cells $(62,64)$ or Treg involvement $(60)$.

AIRE expression levels may indicate an autoimmune disease susceptibility status. Some data in the literature support the idea that the level of AIRE in the thymus is correlated with susceptibility to autoimmune diseases. Thymic Aire expression levels correlate with the expression of TSA genes (65) and also with T cell thymic deletion induced by antigen. Indeed, the loss of 1 copy of Aire significantly reduces the thymic expression of the endogenous insulin gene, resulting in a substantial increase in islet-reactive CD $4^{+}$ T cells escaping thymic deletion and a dramatically increased progression to diabetes (13). Other models, such as collagen-induced arthritis, show that mice heterozygous for Aire exhibit a clinical response that is intermediate between that found in Aire $e^{-/}$and WT mice (59). Our findings clearly support the idea of a direct link between levels of AIRE and susceptibility to autoimmune diseases. Decreasing the level of AIRE specifically in the thymus of male mice led to an increased susceptibility to induced thyroiditis, as assessed by the level of antibodies to thyroglobulin, and the number of infiltrating cells in the thyroid. An additional concept related to this finding has been proposed recently. In nonobese diabetic mice, age-related downregulation of Aire expression in mTECs precedes the emergence of type 1 diabetes mellitus (66). By using a doxycycline-regulated transgene to target Aire expression in mouse mTECs, it has been confirmed that the time window of Aire deficiency (early age) is sufficient to increase susceptibil- 
ity to autoimmune diseases in adults (67). This fact emphasizes that the age-related perturbation of the negative selection process includes a reduced expression of AIRE and an increased autoimmune phenotype in mice (68), thereby validating our observations. We demonstrated that AIRE thymic expression is reduced in females compared with males, which supports females being biased toward a high susceptibility to autoimmune diseases. Furthermore, we also demonstrated that sex differential AIRE expression occurred in mouse strains such as SJL or C57BL/6, which display autoimmune disease susceptibility in females.

In humans, several examples of decreased AIRE expression have been associated with autoimmune diseases: Omenn patients (with a mutation in the RAG gene) have reduced AIRE expression and autoimmune pathology (69). Thymoma patients frequently develop autoimmune diseases and have a defect in AIRE expression (70). A very recent manuscript shows that reduction in AIRE expression in the thymoma is more striking in thymoma patients with autoimmune diseases than in thymoma patients without autoimmune diseases (71). Furthermore, Down syndrome (DS) patients are highly susceptible to autoimmune diseases (72), and a quantification of the number of $\mathrm{AIRE}^{+}$cells in the thymus of a Brazilian cohort of DS patients revealed a 2-fold reduced number of $\mathrm{AIRE}^{+}$cells compared with that in controls (73). Interestingly, in an independent recent study from a Spanish cohort, similar results were observed. When the authors looked specifically at the levels of AIRE in the thymus of the individuals who had developed hypothyroidism in the course of 20 years, the reduction (about 2-fold) in intrathymic AIRE expression was greater in DS individuals with hypothyroidism than in those without hypothyroidism. These data suggest that moderate changes in AIRE expression (and TSAs) can have a major impact on predisposition to autoimmunity (17).

Of note, patients presenting type 1 autoimmune polyglandular syndrome due to a genetic AIRE deficiency are similarly represented among males and females (74), emphasizing that susceptibility to autoimmune disease sexual dimorphism disappears in the absence of functional AIRE. Together, these data from the literature along with our findings support a relationship between the level of AIRE and susceptibility to autoimmune diseases.

Physiological links among AIRE, estrogens, and autoimmunity. Our results contribute another piece of evidence to this AIRE/tolerance/autoimmune disease susceptibility puzzle (Figure 7). Our data support the idea that the main female sexual hormone, estrogen, downregulates the expression of AIRE in mTECs, increasing female susceptibility to autoimmune diseases. Interestingly, DHT increases the expression of AIRE, and experiments with both hormones show that the balance between estrogen and DHT affects levels of AIRE, with estrogen reducing it and DHT increasing it, although the estrogen effect was dominant when both hormones were added at $10^{-9} \mathrm{M}$. In addition, the mechanisms of action are dissimilar: estrogen clearly acts by methylation of AIRE while DHT does not affect it. It is likely that DHT effects are mediated via an indirect mechanism through modulation of expression and/ or function of molecules contributing to the transduction pathway involved in AIRE expression. Estrogen levels rise during puberty, the period when female predisposition to autoimmune diseases is very high. Even though females during this period exhibit an enhanced immune capability, with stronger humoral and cel- lular immune response compared with males, they also tend to overreact to autoantigens and then become more susceptible to autoimmune diseases (7). Estrogen regulates different processes of the immune response, such as immune cell differentiation, cytokine production, cytokine receptor expression, and regulation of $\mathrm{Ca}^{2+}(5,7)$. Even the regulation of sex differential gene expression in immune cells after puberty in mice is mediated through the estrogen pathway (75-77). These estrogen effects are mediated through 2 nuclear receptors, ER $\alpha$ and ER $\beta$, both expressed at various levels in most immune cells ( $\mathrm{T}$ cells, TECs, dendritic cells, B cells). Using castrated mice and ER $\alpha$-deficient mice, we demonstrated that estrogen, through $\mathrm{ER} \alpha$, affects mTEC function by reducing the expression of AIRE and then decreasing the level of TSAs involved in the tolerance process. Therefore, sexual hormones not only affect immune effector functions, but also act at an upstream level by modifying levels of AIRE. This process could have a pronounced consequence upon the negative selection of autoreactive cells. This hypothesis is supported by our data showing that the deleterious effect of estrogen on the autoimmune response is linked to the thymus. Although neonatal thymectomy is often associated with increased autoimmunity due to a defect in expansion and differentiation of Tregs $(78,79)$, the hormonal environment in specific conditions of timing and mouse strain could induce differential effects in the absence or the presence of the thymus. Indeed, our data show that, while estrogen increased antibodies to thyroglobulin in nonthymectomized mice, it had no effect when the mice were thymectomized, indicating that the deleterious effect of estrogen was essentially a central effect. In support of this hypothesis, similar findings were shown in several models of autoimmunity. First, in the model of PVG/c rats, estrogen administration resulted in partial suppression of thyroiditis in thymectomized and irradiated rats (80). Second, in the model of glomerulonephritis in NZB/NZW FI mice, estrogen increased the level of antibodies to poly A, but neonatal thymectomy abolished the overproduction of these antibodies (81).

Taking into account the links among estrogen, AIRE, thymus, and autoimmunity, one could expect the number of peripheral autoreactive cells to be higher in females than in males. Indeed, in experimental lupus-prone mice, the number of pathogenic reactive $\mathrm{CD}^{+}$splenic $\mathrm{T}$ cells was much higher in females than in males (82). In addition, data from over 500 individuals showed that females displayed a higher frequency of increased $\mathrm{T}$ cell reactivity to immunodominant PLP peptides compared with males, particularly in individuals who do not have multiple sclerosis (MS) (83). These data could be explained by a lower expression of AIRE in the thymus of females compared with males. Combined with a higher number of thymic emigrants in females (84), the risk of developing autoimmune disease is therefore much higher in females than males.

In conclusion, our data provide another line of evidence in the complex picture of female sex-associated susceptibility bias to autoimmune diseases (Figure 7). We emphasize the role of female sex hormones. During puberty, estrogen downregulates thymic AIRE expression and may reduce the efficiency of the tolerance process regulated by AIRE, resulting in increased export of autoreactive cells compared with that in males. The equilibrium between autoreactive $\mathrm{T}$ cells and Tregs is accordingly tenuous and can, in 
predisposed individuals, become unbalanced and result in the development of autoimmune diseases in the presence of a triggering external factor. In industrialized countries, the percentage of patients with autoimmune diseases is on the rise. This prompts an investigation of the role of increased estrogen-like molecules in the environment and phytoestrogen-rich diets.

\section{Methods}

Human samples. Human thymic fragments were obtained from 84 immunologically normal male and female patients ( 5 months to 46 years old) undergoing corrective cardiovascular surgery at the Marie Lannelongue Chirurgical Center (Le Plessis-Robinson, France). The samples were divided into 3 groups: the prepubescent child group (5 months to 11 years old) included 16 females and 15 males; the pubescent child group (13 to 17 years old) included 17 females and 19 males, and the adult group ( $>18$ years old) included 9 female and 12 males. Neonate thymuses (up to 4 months) were not included in the study, since they were shown to display specific hormonal changes (85). All tissue samples were fast-frozen in liquid nitrogen within 30 minutes of their excision from patients. The analysis of AIRE mRNA was performed on 48 thymuses, and counting of $\mathrm{AIRE}^{+}$cells was performed in 36 additional thymuses.

Mice. C57BL/6, SJL, and C3H mice were purchased from Janvier Laboratory. ER $\alpha-\mathrm{KO}$ mice (86), backcrossed on a C57BL/6 background (more than 10 generations), were from R. Habert's mouse colony (CEA, Fontenay-aux-Roses, France; material transfer authorization no. 2010-036, Institut Clinique de la Souris, Strasbourg, France). Male and female mice were 3 to 10 weeks old ( $n \geq 3$ per age and sex). Male and female SJL mice at 5 weeks old were bilaterally castrated and allowed to recover for 2 weeks before being sacrificed for thymus analysis. Male SJL mice at 7 weeks old received a $10-\mu$ intrathymic injection of a vector solution (8 to 10.1012 viral genomes per $\mathrm{ml}$ ) and were allowed to recover for 1 week before being treated for EAT.

The experiment investigating the effect of castration and thymectomy on EAT development is represented in Supplemental Figure 12. In this experiment, male SJL mice at 3 weeks old were first castrated and then allowed to recover for 1 week before being thymectomized. Mice were challenged for EAT 2 weeks after thymectomy. Following the castration surgery, male SJL mice were treated every 2 days with $1 \mu \mathrm{g}$ of estradiol by subcutaneous injection $(80,87)$.

Statistics. Comparisons among groups were performed using 1-way ANOVA when more than 2 groups were compared, and parametric or nonparametric $t$ tests for comparison between 2 groups (Wilcoxon test for paired data, and Mann-Whitney $U$ test for unpaired values). Nonparametric tests were used throughout. $P<$ 0.05 was considered statistically significant. GraphPad prism was used for all statistical analysis.
Study approval. Human studies were approved by the local ethics committee (CPP Kremlin-Bicêtre, France: agreement no. 06-018; CCP Ile de France Paris 6, France: agreement no. C09-36). In accordance with the 2004 French Bioethics Law, informed consent was obtained from patients or their legal representatives. Animal studies were approved by the French Ministry of Agriculture Committee for Animal Use (NDM certificate 75-1817). All surgical procedures were performed in accordance with NDM authorization from the French Ministry of Agriculture (agreement no. 1-63IUTCIFd-Chir-07) for surgery on small rodents. All animals were handled according to the Animal Care and Use of Laboratory Animal guidelines of the French Ministry of Research (study approval no. 02638-02). Refer to the Supplemental Methods for further description of the experimental procedures. All primers used in the study are described in Supplemental Table 6.

\section{Author contributions}

ND designed and performed most of the experiments, analyzed the data, and interpreted the results. JB performed the immunofluorescence and cell counting as well as PCR experiments. GCC performed microarray analysis and initiated the experiments. MGB and MB produced and provided the AIRE silencing RNA. CB and AC performed the FTOC experiments. AS and RN provided human thymic tissues. DK and RLP provided helpful suggestions for designing experiments. SBA was involved in all aspects of the study, including design, data analysis, and interpretation of the results. ND and SBA wrote the manuscript. All authors discussed the results and commented on the manuscript.

\section{Acknowledgments}

We would like to thank M. Attia, P. Cufi, S. Dessolin, and A. Gradolatto for their help and advice on some of the experiments. We would like to acknowledge the Flow Cytometry Core (CyPS) and the Centre d'Expérimentation Fonctionnelle (CEF) of Pierre and Marie Curie University for their advice. We would like to thank Nathalie Mougenot for her help and advice for the thymectomy procedure. We thank M. Kanovsky for editing the manuscript. This work was supported by MYASTAID (LSHM-CT-2006-037833) and FIGHT-MG (HEALTH-2009-242-210) grants from the European Community and a grant from the Association Française contre les Myopathies obtained by S. Berrih-Aknin. This manuscript is dedicated to D. Emilie, who died accidentally in February 2011, for his support and encouragement at the initiation of this work.

Address correspondence to: Sonia Berrih-Aknin, Université Pierre et Marie Curie - Paris VI, INSERM U974- Hôpital La Pitié Salpêtrière, 105 Bd de l'hôpital, 75013 Paris, France. Phone: 00.33.0.1.40.77.81.28; E-mail: sonia.berrih-aknin@upmc.fr.
1. Dai R, Ahmed SA. Sexual dimorphism of miRNA expression: a new perspective in understanding the sex bias of autoimmune diseases. Ther Clin Risk Manag. 2014;10:151-163.

2. Anaya JM. Common mechanisms of autoimmune diseases (the autoimmune tautology). Autoimmun Rev. 2012;11(11):781-784.

3. Geurs TL, Hill EB, Lippold DM, French AR. Sex differences in murine susceptibility to systemic viral infections. J Autoimmun.
2012;38(2-3):J245-J253.

4. Berrih-Aknin S. Myasthenia Gravis: paradox versus paradigm in autoimmunity. JAutoimmun. 2014;52:1-28.

5. Gonzalez DA, Diaz BB, Rodriguez Perez Mdel C, Hernandez AG, Chico BN, de Leon AC. Sex hormones and autoimmunity. Immunol Lett. 2010;133(1):6-13.

6. Hill L, Jeganathan V, Chinnasamy P, Grimaldi C, Diamond B. Differential roles of estrogen recep- tors $\alpha$ and $\beta$ in control of $\mathrm{B}$-cell maturation and selection. Mol Med. 2011;17(3-4):211-220.

7. Cutolo M, Sulli A, Straub RH. Estrogen metabolism and autoimmunity. Autoimmun Rev. 2012;11(6-7):A460-464.

8. Kyewski B, Klein L. A central role for central tolerance. Annu Rev Immunol. 2006;24:571-606.

9. Hinterberger M, Aichinger M, Prazeres da Costa O, Voehringer D, Hoffmann R, Klein L. Autonomous role of medullary thymic epithelial cells in 
central CD4(+) T cell tolerance. Nat Immunol. 2010;11(6):512-519.

10. Ishii T, Suzuki Y, Ando N, Matsuo N, Ogata T. Novel mutations of the autoimmune regulator gene in two siblings with autoimmune polyendocrinopathy-candidiasis-ectodermal dystrophy. JClin Endocrinol Metab. 2000;85(8):2922-2926.

11. Ahonen P, Myllarniemi S, Sipila I, Perheentupa J. Clinical variation of autoimmune polyendocrinopathy-candidiasis-ectodermal dystrophy (APECED) in a series of 68 patients. N Engl JMed. 1990;322(26):1829-1836.

12. Anderson MS, et al. Projection of an immunological self shadow within the thymus by the aire protein. Science. 2002;298(5597):1395-1401.

13. Liston A, et al. Gene dosage - limiting role of Aire in thymic expression, clonal deletion, and organ-specific autoimmunity. J Exp Med. 2004;200(8):1015-1026.

14. Taniguchi RT, et al. Detection of an autoreactive $\mathrm{T}$-cell population within the polyclonal repertoire that undergoes distinct autoimmune regulator (Aire)-mediated selection. Proc Natl Acad Sci U S A. 2012;109(20):7847-7852.

15. Kuroda N, et al. Development of autoimmunity against transcriptionally unrepressed target antigen in the thymus of Aire-deficient mice. JImmunol. 2005;174(4):1862-1870.

16. Hubert FX, et al. Aire-deficient C57BL/6 mice mimicking the common human 13-base pair deletion mutation present with only a mild autoimmune phenotype. J Immunol. 2009;182(6):3902-3918.

17. Gimenez-Barcons M, et al. Autoimmune predisposition in Down syndrome may result from a partial central tolerance failure due to insufficient intrathymic expression of AIRE and peripheral antigens. J Immunol. 2014;193(8):3872-3879.

18. Bonfanti P, Claudinot S, Amici AW, Farley A, Blackburn CC, Barrandon Y. Microenvironmental reprogramming of thymic epithelial cells to skin multipotent stem cells. Nature. 2010;466(7309):978-982.

19. Dooley J, Erickson M, Farr AG. Alterations of the medullary epithelial compartment in the Aire-deficient thymus: implications for programs of thymic epithelial differentiation. JImmunol. 2008;181(8):5225-5232.

20. Arbel-Goren R, Tal A, Stavans J. Phenotypic noise: effects of post-transcriptional regulatory processes affecting mRNA. Wiley Interdiscip Rev RNA. 2014;5(2):197-207.

21. Christadoss P, Poussin M, Deng C. Animal models of myasthenia gravis. Clin Immunol. 2000;94(2):75-87.

22. Yu CY, Whitacre CC. Sex, MHC and complement C4 in autoimmune diseases. Trends Immunol. 2004;25(12):694-699.

23. Alderuccio F, Sentry JW, Marshall AC, Biondo $\mathrm{M}$, Toh BH. Animal models of human disease: experimental autoimmune gastritis--a model for autoimmune gastritis and pernicious anemia. Clin Immunol. 2002;102(1):48-58.

24. Gray DH, et al. Unbiased analysis, enrichment and purification of thymic stromal cells. JImmunol Methods. 2008;329(1-2):56-66.

25. Kont $\mathrm{V}$, et al. DNA methylation signatures of the AIRE promoter in thymic epithelial cells, thymomas and normal tissues. Mol Immunol. 2011;49(3):518-526.

26. Giraud M, et al. An IRF8-binding promoter variant and AIRE control CHRNA1 promiscuous expression in thymus. Nature. 2007;448(7156):934-937.

27. Taubert R, Schwendemann J, Kyewski B. Highly variable expression of tissue-restricted selfantigens in human thymus: implications for self-tolerance and autoimmunity. Eur J Immunol. 2007;37(3):838-848.

28. Aricha R, Feferman T, Scott HS, Souroujon MC, Berrih-Aknin S, Fuchs S. The susceptibility of Aire(-/-) mice to experimental myasthenia gravis involves alterations in regulatory T cells. J Autoimmun. 2011;36(1):16-24.

29. Misharin AV, Nagayama Y, Aliesky HA, Rapoport B, McLachlan SM. Studies in mice deficient for the autoimmune regulator (Aire) and transgenic for the thyrotropin receptor reveal a role for Aire in tolerance for thyroid autoantigens. Endocrinology. 2009;150(6):2948-2956.

30. Fernando R, Lu Y, Atkins SJ, Mester T, Branham K, Smith TJ. Expression of thyrotropin receptor, thyroglobulin, sodium-iodide symporter, and thyroperoxidase by fibrocytes depends on AIRE. J Clin Endocrinol Metab. 2014;99(7):E1236-E1244.

31. Derbinski J, et al. Promiscuous gene expression in thymic epithelial cells is regulated at multiple levels. J Exp Med. 2005;202(1):33-45.

32. Fornari TA, Donate PB, Macedo C, Marques MM, Magalhaes DA, Passos GA. Age-related deregulation of Aire and peripheral tissue antigen genes in the thymic stroma of non-obese diabetic (NOD) mice is associated with autoimmune type 1 diabetes mellitus (DM-1). Mol Cell Biochem. 2010;342(1-2):21-28.

33. Calder AE, Hince MN, Dudakov JA, Chidgey AP, Boyd RL. Thymic involution: where endocrinology meets immunology. Neuroimmunomodulation. 2011;18(5):281-289.

34. Heng TS, Goldberg GL, Gray DH, Sutherland JS, Chidgey AP, Boyd RL. Effects of castration on thymocyte development in two different models of thymic involution. J Immunol. 2005;175(5):2982-2993.

35. Hince M, Sakkal S, Vlahos K, Dudakov J, Boyd R, Chidgey A. The role of sex steroids and gonadectomy in the control of thymic involution. Cell Immunol. 2008;252(1-2):122-138.

36. Dooley J, Liston A. Molecular control over thymic involution: from cytokines and microRNA to aging and adipose tissue. Eur J Immunol. 2012;42(5):1073-1079.

37. Fitzpatrick FT, Kendall MD, Wheeler MJ, Adcock IM, Greenstein BD. Reappearance of thymus of ageing rats after orchidectomy. JEndocrinol. 1985;106(3):R17-R19.

38. Simpson ER. Sources of estrogen and their importance. J Steroid Biochem Mol Biol. 2003;86(3-5):225-230.

39. Zhao H, Tian Z, Hao J, Chen B. Extragonadal aromatization increases with time after ovariectomy in rats. Reprod Biol Endocrinol. 2005;3:6.

40. Nazzal D, Gradolatto A, Truffault F, Bismuth J, Berrih-Aknin S. Human thymus medullary epithelial cells promote regulatory T-cell generation by stimulating interleukin-2 production via ICOS ligand. Cell Death Dis. 2014;5:e1420.

41. Cufi P, et al. Implication of double-stranded RNA signaling in the etiology of autoimmune myasthenia gravis. Ann Neurol. 2013;73(2):281-293.

42. Nancy P, Berrih-Aknin S. Differential estrogen receptor expression in autoimmune myasthenia gravis. Endocrinology. 2005;146(5):2345-2353.

43. Yellayi S, et al. Normal development of thymus in male and female mice requires estrogen/estrogen receptor-alpha signaling pathway. Endocrine. 2000;12(3):207-213.

44. Murumagi A, Vahamurto P, Peterson P. Characterization of regulatory elements and methylation pattern of the autoimmune regulator (AIRE) promoter. J Biol Chem. 2003;278(22):19784-19790.

45. Metzger TC, Anderson MS. Control of central and peripheral tolerance by Aire. Immunol Rev. 2011;241(1):89-103.

46. Vladutiu AO, Kenney EM. Thyroid function in mice with experimental autoimmune thyroiditis. Clin Exp Immunol. 1985;61(2):257-264.

47. Hatzioannou A, Liakata E, Karras E, Thrasyvoulides A, Alevizaki M, Lymberi P. Pathogenicity of a human thyroglobulin peptide (2340-2359) in mice with high or low genetic susceptibility to thyroiditis. Immunology. 2007;122(3):343-349.

48. Okayasu I, Kong YM, Rose NR. Effect of castration and sex hormones on experimental autoimmune thyroiditis. Clin Immunol Immunopathol. 1981;20(2):240-245.

49. Jabed A, Wagner S, McCracken J, Wells DN, Laible G. Targeted microRNA expression in dairy cattle directs production of beta-lactoglobulinfree, high-casein milk. Proc Natl Acad Sci U S A. 2012;109(42):16811-16816.

50. Duque $S$, et al. Intravenous administration of self-complementary AAV9 enables transgene delivery to adult motor neurons. Mol Ther. 2009;17(7):1187-1196.

51. Giron-Gonzalez JA, et al. Consistent production of a higher TH1:TH2 cytokine ratio by stimulated T cells in men compared with women. Eur J Endocrinol. 2000;143(1):31-36.

52. Kanda N, Tamaki K. Estrogen enhances immunoglobulin production by human PBMCs. JAllergy Clin Immunol. 1999;103(2 pt 1):282-288.

53. Mo R, Chen J, Grolleau-Julius A, Murphy HS, Richardson BC, Yung RL. Estrogen regulates CCR gene expression and function in T lymphocytes. J Immunol. 2005;174(10):6023-6029.

54. Bonita RE, Rose NR, Rasooly L, Caturegli P, Burek CL. Kinetics of mononuclear cell infiltration and cytokine expression in iodine-induced thyroiditis in the NOD-H2h4 mouse. Exp Mol Pathol. 2003;74(1):1-12.

55. Caturegli P, et al. Anatabine ameliorates experimental autoimmune thyroiditis. Endocrinology. 2012;153(9):4580-4587.

56. Forster R, Davalos-Misslitz AC, Rot A. CCR7 and its ligands: balancing immunity and tolerance. Nat Rev Immunol. 2008;8(5):362-371.

57. DeVoss JJ, et al. An autoimmune response to odorant binding protein 1a is associated with dry eye in the Aire-deficient mouse. JImmunol. 2010;184(8):4236-4246.

58 . Wada N, et al. Aire-dependent thymic expression of desmoglein 3 , the autoantigen in pemphigus 
vulgaris, and its role in T-cell tolerance. J Invest Dermatol. 2011;131(2):410-417.

59. Campbell IK, et al. Autoimmune regulator controls $\mathrm{T}$ cell help for pathogenetic autoantibody production in collagen-induced arthritis. Arthritis Rheum. 2009;60(6):1683-1693.

60. Pomie C, et al. Autoimmune regulator (AIRE)deficient $\mathrm{CD} 8+\mathrm{CD} 28$ low regulatory $\mathrm{T}$ lymphocytes fail to control experimental colitis. Proc Natl Acad Sci U S A. 2011;108(30):12437-12442.

61. Aharoni R, et al. Age dependent course of EAE in Aire ${ }^{-/}$mice. J Neuroimmunol. 2013;262(1-2):27-34.

62. Kurisaki H, Nagao Y, Nagafuchi S, Mitsuyama M. Autoimmune gastro-pancreatitis with antiprotein disulfide isomerase-associated 2 autoantibody in Aire-deficient BALB/cAnN mice. PLoS One. 2013;8(8):e73862.

63. Niki S, et al. Alteration of intra-pancreatic targetorgan specificity by abrogation of Aire in NOD mice. JClin Invest. 2006;116(5):1292-1301.

64. Yeh S, et al. Spontaneous T cell mediated keratoconjunctivitis in Aire-deficient mice. Br JOphthalmol. 2009;93(9):1260-1264.

65. Kont V, Laan M, Kisand K, Merits A, Scott HS, Peterson P. Modulation of Aire regulates the expression of tissue-restricted antigens. $\mathrm{Mol}$ Immunol. 2008;45(1):25-33.

66. Oliveira EH, et al. Expression profile of peripheral tissue antigen genes in medullary thymic epithelial cells (mTECs) is dependent on mRNA levels of autoimmune regulator (Aire). Immunobiology. 2013;218(1):96-104.

67. Guerau-de-Arellano M, Martinic M, Benoist C, Mathis D. Neonatal tolerance revisited: a perinatal window for Aire control of autoimmunity. JExp Med. 2009;206(6):1245-1252.

68. Xia J, Wang H, Guo J, Zhang Z, Coder B, Su DM. Age-related disruption of steady-state thymic medulla provokes autoimmune phenotype via perturbing negative selection. Aging Dis. 2012;3(3):248-259.

69. Cavadini P, et al. AIRE deficiency in thymus of 2 patients with Omenn syndrome. JClin Invest. 2005;115(3):728-732.

70. Marx A, et al. The autoimmune regulator AIRE in thymoma biology: autoimmunity and beyond. J Thorac Oncol. 2010;5(10 suppl 4):S266-S272.

71. Liu Y, et al. Autoimmune regulator expression in thymomas with or without autoimmune disease. Immunol Lett. 2014;161(1):50-56.

72. Goldacre MJ, Wotton CJ, Seagroatt V, Yeates D. Cancers and immune related diseases associated with Down's syndrome: a record linkage study. Arch Dis Child. 2004;89(11):1014-1017.

73. Lima FA, et al. Decreased AIRE expression and global thymic hypofunction in Down syndrome. JImmunol. 2011;187(6):3422-3430.

74. Kahaly GJ. Polyglandular autoimmune syndromes. Eur JEndocrinol. 2009;161(1):11-20.

75. Stygar D, Masironi B, Eriksson H, Sahlin L. Studies on estrogen receptor (ER) alpha and beta responses on gene regulation in peripheral blood leukocytes in vivo using selective ER agonists. JEndocrinol. 2007;194(1):101-119.

76. Panchanathan R, Liu H, Choubey D. Expression of murine Unc $93 \mathrm{~b} 1$ is up-regulated by interferon and estrogen signaling: implications for sex bias in the development of autoimmunity. Int Immunol. 2013;25(9):521-529.

77. Lu Y, et al. Modulation of the expression of the transcription factors T-bet and GATA-3 in immortalized human endometrial stromal cells (HESCs) by sex steroid hormones and cAMP. Reprod Sci. 2013;20(6):699-709.

78. Samy ET, Wheeler KM, Roper RJ, Teuscher C, Tung KS. Cutting edge: Autoimmune disease in day 3 thymectomized mice is actively controlled by endogenous disease-specific regulatory $\mathrm{T}$ cells. JImmunol. 2008;180(7):4366-4370.

79. Yamada A, et al. Impaired expansion of regulatory $\mathrm{T}$ cells in a neonatal thymectomy-induced autoimmune mouse model. Am J Pathol. 2015;185(11):2886-2897.

80. Ansar Ahmed S, Young PR, Penhale WJ. The effects of female sex steroids on the development of autoimmune thyroiditis in thymectomized and irradiated rats. Clin Exp Immunol. 1983;54(2):351-358.

81. Roubinian JR, Talal N, Greenspan JS, Goodman JR, Siiteri PK. Effect of castration and sex hormone treatment on survival, anti-nucleic acid antibodies, and glomerulonephritis in NZB/ NZW F1 mice. J Exp Med. 1978;147(6):1568-1583.

82. Feng F. The role of estrogen in the induction and modulation of systemic lupus erythromatosus (SLE) [doctoral thesis]. Ithica, New York: Cornell University; 2007.

83. Greer JM, Csurhes PA, Pender MP, McCombe PA. Effect of gender on T-cell proliferative responses to myelin proteolipid protein antigens in patients with multiple sclerosis and controls. J Autoimmun. 2004;22(4):345-352.

84. Pido-Lopez J, Imami N, Aspinall R. Both age and gender affect thymic output: more recent thymic migrants in females than males as they age. Clin Exp Immunol. 2001;125(3):409-413.

85. Rey RA. Mini-puberty and true puberty: differences in testicular function. Ann Endocrinol (Paris). 2014;75(2):58-63.

86. Dupont S, Krust A, Gansmuller A, Dierich A, Chambon P, Mark M. Effect of single and compound knockouts of estrogen receptors alpha $(\mathrm{ER} \alpha)$ and beta $(\mathrm{ER} \beta)$ on mouse reproductive phenotypes. Development. 2000;127(19):4277-4291.

87. Ansar Ahmed S, Penhale WJ, Talal N. Sex hormones, immune responses, and autoimmune diseases. Am J Pathol. 1985;121(3):531-551. 